\title{
Lau Effect Using LED Array for Lithography
}

\author{
Xinrui Cao ${ }^{1}\left[\right.$ Patrick Feßer $^{1} \cdot$ Stefan Sinzinger ${ }^{1}$
}

Received: 15 December 2020 / Revised: 4 May 2021 / Accepted: 10 May 2021 / Published online: 4 June 2021

(c) The Author(s) 2021

\begin{abstract}
Illumination with LEDs is of increasing interest in imaging and lithography. In particular, compared to lasers, LEDs are temporally and spatially incoherent, so that speckle effects can be avoided by the application of LEDs. Besides, LED arrays are qualified due to their high optical output power. However, LED arrays have not been widely used for investigating optical effects, e.g., the Lau effect. In this paper, we propose the application of an LED array for realizing the Lau effect by taking into account the influence of the coherence properties of illumination on the Lau effect. Using spatially incoherent illumination with the LED array or a single LED, triangular distributed Lau fringes can be obtained. We apply the obtained Lau fringes in the optical lithography to produce analog structures. Compared to a single LED, the Lau fringes using the LED array have significantly higher intensities. Hence, the exposure time in the lithography process is largely reduced.
\end{abstract}

Keywords Lau effect $\cdot$ LED array $\cdot$ Lithography $\cdot$ Spatial coherence properties of illumination

\section{Introduction}

The Lau effect was discovered by the German physicist Ernst Lau in 1948 [1]. In the setup of the Lau's experiment, two identical line gratings are located one behind the other. With the illumination from an extended white light source on the first grating, colored fringes were observed at infinity for some grating separations. Jahns and Lohmann [2] derived the mathematical description of the Lau effect by using the scalar diffraction theory. The intensity detected in the observation plane can be written as the cross-correlation between the amplitude square of the first grating and the amplitude square of the Fresnel transform of the second grating. It is known that the cross-correlation of two rectangles is a triangle. Therefore, when two periodic rectangular amplitude gratings are located with a proper distance in the setup of the Lau's experiment, periodic triangular distributed intensities can be obtained in the observation plane. Sudol and Thompson [3] interpreted the Lau effect with coherence theory and the cross-spectral density function. Liu [4] and Tu and Zhan [5] used the ambiguity function to analyze fringe

Xinrui Cao

xinrui.cao@tu-ilmenau.de

1 Fachgebiet Technische Optik, Institut für Mikro- und Nanotechnologien (IMN) MacroNano®, Technische

Universität Ilmenau, Ilmenau, Germany characteristics under spatially partially coherent illumination as well as the two extremes. The obtained intensity distributions varied with the change of the spatial coherence properties of illumination. All the aforementioned publications verify that spatially incoherent illumination is required to obtain Lau fringes.

Spatially incoherent illumination can be provided not only by an extended light source, e.g., a mercury arc lamp or an LED, but also an LED array. In the application of mask aligner lithography, an LED is qualified due to its low electrical power consumption, long lifetime, and free and easy switchability, etc. [6, 7]. An LED array is more advantageous than a single LED due to its high output optical power [8], saving exposure time such that the disturbance from the environment is reduced.

Our goal is to use Lau fringes to obtain periodic triangular distributed structures in optical lithography. After demonstrating the potential of the Lau effect for lithographic applications, we propose to use an LED array to realize the Lau effect and to reduce the exposure time in the lithography process. In Sect. 3, we derive a simulation model to study the influence of spatial coherence properties of illumination on creating the Lau fringes. Our results indicate the importance of various illumination angles for the Lau effect. In Sect. 4, we present the proposed illumination concept using an LED array for the Lau effect. With this concept, spatially incoherent illumination is ensured. In Sect. 5, we compare 
the lithographically illuminated structures that are exposed by using the LED array and a single LED. In Sect. 6, we conclude that the LED array is superior to a single LED in saving the exposure time in optical lithography. Moreover, we look forward to wider applications of LED arrays in optical systems, e.g., the image of the LED array as a structure illumination.

\section{Lau Effect for Optical Lithography}

In optical lithography, light, i.e., electromagnetic radiation is used for patterning structures on photosensitive material (photoresist) [9]. Besides mask projection or direct laser writing, optical phenomena, e.g., the self-images of the object in the Talbot effect [10] and the interference, e.g., from two coherent light beams that is based on the Young's double-slit experiment [11, 12], have already been used for producing periodic structures in optical lithography. Compared to direct laser lithography, e.g., single-photon [13] or two-photon polymerization [14, 15], these approaches provide an intensity distribution extending over large areas and thus allow for parallel structuring to save exposure time in the lithography process.

In the above-mentioned examples of direct laser lithography and optical phenomena for lithography, lasers are applied as light sources because of their high degree of coherence and high optical power density. Collimated illumination with a laser can be used to directly illuminate objects with periodic structures, e.g., simple line gratings or grating with two-dimensional structures [16]. The selfimages of the gratings with the same or demagnified periods can be employed in optical lithography to produce periodic patterns on photoresist. However, due to the coherence of the collimated laser, speckles appear and influence the quality of the lithographic structures $[17,18]$. In order to minimize the speckle effect, various techniques have been applied, e.g., multiple exposure [19], or the use of a rotating, holographic diffuser [20] to reduce the spatial coherence of the laser light.

Another optical phenomenon, i.e., the Lau effect, which works with spatially incoherent light, can also be applied in optical lithography [21]. The Lau effect differs from the Talbot effect in the spatial coherence properties of the illumination, the number of gratings, and in the aimed observation planes. The Talbot effect is the light propagation of the single grating in the near field using spatially coherent illumination [22, 23]. The adding of spatially coherent illuminations with different angles changes the illumination into spatially incoherent illumination. Hence, the corresponding intensities of the Talbot carpets of the grating under different illumination angles are summed up. A high lateral resolution of the Talbot carpets is achieved
[24]. For the Lau effect, two gratings are used. The Lau effect is the light propagation of the modulated second grating in the far field, i.e., in the back focal plane of the Fourier lens that is located behind the second grating, when spatially incoherent illumination is used. When two identical rectangular amplitude Ronchi gratings are used and the second grating is located at one half of the Talbot distance behind the first grating, the intensity distribution of the Lau fringes does not correspond to the self-images of the gratings, but results as the cross-correlation between the amplitude square of the first grating and the amplitude square of the self-image of the second grating, e.g., a periodic triangular distribution for rectangular gratings. Compared to the rectangular distribution, the triangular distribution has a continuous change of the intensity width. This can lead to different widths of the lithographed structures. The peak of the triangular distribution represents the resolution of structures when a grayscale photoresist is used for the exposure in the lithography process. Speckle effects are avoided by the application of incoherent light sources, e.g., LEDs.

It is known that a small oblique illumination angle leads to both lateral and axial shifts of the self-images in the Talbot effect [25]. These shifts reduce the symmetry of the self-images of the rectangular or sinusoidal gratings in each period. Similarly, illumination angles also affect the intensity distribution in the setup of the Lau experiment.

\section{Influence of Spatial Coherence Properties of Illumination on the Lau Effect}

Two fundamental models of illumination with different spatial coherence properties [26] are plane wave and Köhler illumination, which are shown in Fig. 1. Plane wave illumination can be understood as collimated light from a single point source. Therefore, it is strictly spatially coherent. Köhler illumination is generally assumed to be spatially incoherent. In the Köhler illumination, the image of the extended light source is magnified and located in the front focal plane of the condenser. Therefore, the object is illuminated by many plane waves from different angles, which correspond to different point sources.

Plane wave and Köhler illumination lead to different intensity distributions in the observation plane in the setup of Lau's experiment (Fig. 2). To study their differences, we derive the mathematical model of the spatial coherence analysis of light propagation from the first grating to the observation plane, i.e., the back focal plane of the Fourier lens.

The plane wave $U_{\text {illu }}^{\prime}\left(x^{\prime}, y^{\prime}, 0\right)$ is described by the Eq. (1). It has a unit amplitude 1 and a direction cosine $(\cos \alpha, \cos \beta, \cos \gamma)$. 
(a)

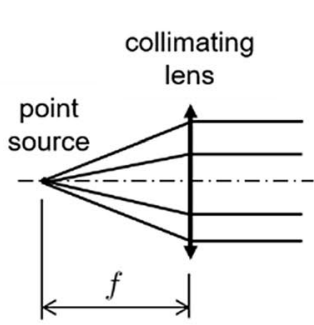

(b)

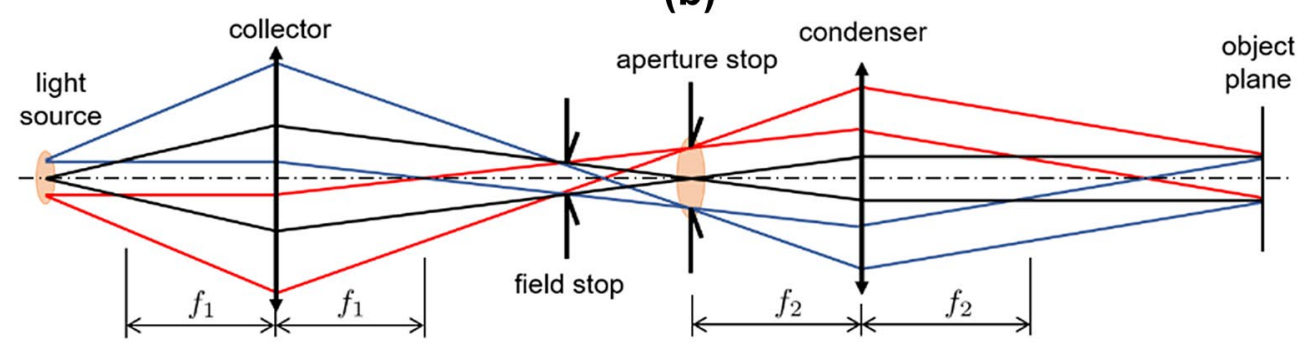

Fig. 1 Two fundamental models of illumination: a plane wave and b Köhler illumination

Fig. 2 Schematic setup with plane wave for studying Lau effect (a)

direction cosine of the illumination $(\cos \alpha, \cos \beta, \cos \gamma)$

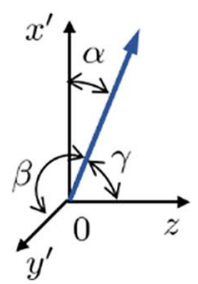

(b) cross section at $y=0$

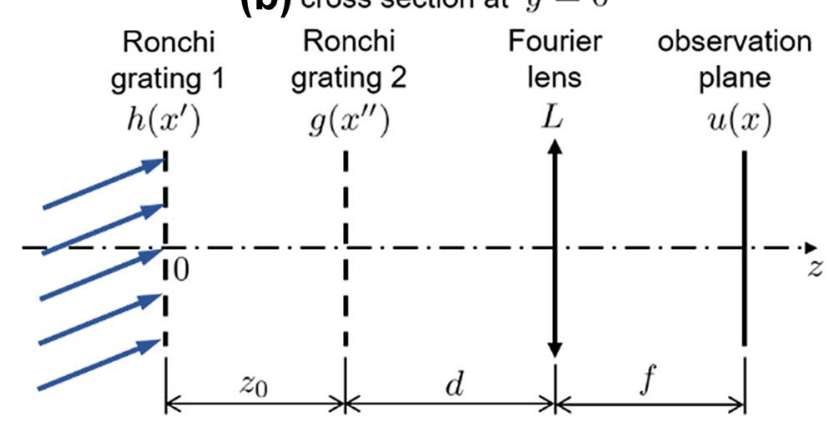

$U_{\text {illu }}^{\prime}\left(x^{\prime}, y^{\prime}, 0\right)=1 \cdot \mathrm{e}^{\mathrm{j}\left(x^{\prime} \cdot \cos \alpha+y^{\prime} \cdot \cos \beta+z \cdot \cos \gamma\right)}=\mathrm{e}^{\mathrm{j}\left(x^{\prime} \cdot \cos \alpha+y^{\prime} \cdot \cos \beta\right)}$

The first Ronchi grating $h\left(x^{\prime}, y^{\prime}, 0\right)$ and the second Ronchi grating $g\left(x^{\prime \prime}, y^{\prime \prime}, z_{0}\right)$ can be described using Fourier series, which are shown in Eqs. (2) and (3), where $A_{m}$ and $A_{n}$ stand for the Fourier coefficients of both periodic binary amplitude gratings, $m$ and $n$ are the indices, and $p_{1}$ and $p_{2}$ are the periods of the gratings.

$h\left(x^{\prime}, y^{\prime}, 0\right)=\sum_{m=-\infty}^{\infty} A_{m} \cdot \mathrm{e}^{\mathrm{j} 2 \pi m \frac{1}{p_{1}} x^{\prime}}$
$g\left(x^{\prime \prime}, y^{\prime \prime}, z_{0}\right)=\sum_{n=-\infty}^{\infty} A_{n} \cdot \mathrm{e}^{\mathrm{j} 2 \pi n \frac{1}{p_{2}}\left(x^{\prime \prime}-\frac{p_{1}}{2}\right)}$
The light propagation $U^{\prime \prime(-)}\left(x^{\prime \prime}, y^{\prime \prime}, z_{0}\right)$ from the first grating to the second grating is derived in Eq. (4) by using the Fresnel propagation [27]. It can be seen that the variation of the direction cosine $(\cos \alpha, \cos \beta, \cos \gamma)$ of the illumination, i.e., the illumination angle, results in both lateral and axial shifts of the self-imaging of the first grating [25].

$$
\begin{aligned}
U^{\prime \prime(-)}\left(x^{\prime \prime}, y^{\prime \prime}, z_{0}\right)= & \frac{\mathrm{e}^{\mathrm{j} \frac{2 \pi}{\lambda} z_{0}}}{\mathrm{j} \lambda z_{0}} \cdot \iint_{-\infty}^{\infty} U_{\mathrm{illu}}^{\prime} \cdot h \cdot \mathrm{e}^{\frac{\mathrm{j} \pi}{\lambda z_{0}}\left[\left(x^{\prime \prime}-x^{\prime}\right)^{2}+\left(y^{\prime \prime}-y^{\prime}\right)^{2}\right]} \mathrm{d} x^{\prime} \mathrm{d} y^{\prime} \\
= & \mathrm{e}^{\mathrm{j} \frac{2 \pi}{\lambda} z_{0}} \cdot \mathrm{e}^{-\mathrm{j} \frac{\pi}{\lambda}\left(\cos ^{2} \alpha+\cos ^{2} \beta\right) z_{0}} \cdot \mathrm{e}^{\mathrm{j} \frac{2 \pi}{\lambda}\left(x^{\prime \prime} \cdot \cos \alpha+y^{\prime \prime} \cdot \cos \beta\right)} \\
& \cdot \sum_{m=-\infty}^{\infty} A_{m} \cdot \mathrm{e}^{-\mathrm{j} \pi\left(m \frac{1}{p_{1}}\right)^{2} \lambda z_{0}} \cdot \mathrm{e}^{\mathrm{j} 2 \pi m \frac{1}{p_{1}} x^{\prime \prime}} \cdot \mathrm{e}^{-\mathrm{j} \pi 2 m \frac{1}{p_{1}} z_{0} \cdot \cos \alpha}
\end{aligned}
$$

The light propagation $U(x, y, z)$ from the second grating to the observation plane, i.e., the back focal plane of the Fourier lens, is derived as the 2-D Fourier transform with a phase shift [28], i.e.,

$U(x, y, z)=\frac{\mathrm{e}^{\mathrm{j} \frac{2 \pi}{\lambda}(d+f)}}{\mathrm{j} \lambda f} \cdot \mathrm{e}^{\mathrm{j} \frac{\pi}{\partial f}\left(1-\frac{d}{f}\right)\left(x^{2}+y^{2}\right)} \cdot F\left\{U^{\prime \prime(-)}\left(x^{\prime \prime}, y^{\prime \prime}, z_{0}\right) \cdot g\left(x^{\prime \prime}, y^{\prime \prime}, z_{0}\right)\right\}$, 
where $f$ and $d$ denote the focal length of the Fourier lens and the distance between the second Ronchi grating and the Fourier lens, respectively.

In our numerical simulations, the plane wave is assumed to have uniform normalized intensity and monochromatic properties (wavelength $\lambda=400 \mathrm{~nm}$ ). Two identical Ronchi gratings have periods $p_{1}=p_{2}=200 \mu \mathrm{m}$ and mark-space ration $\delta_{1}=\delta_{2}=15 \%$. The grating separation is one half of the Talbot distance, i.e., $z_{0}=p_{1}^{2} / \lambda=100 \mathrm{~mm}$. The slits of both Ronchi gratings are parallel to the $y^{\prime}$-axis but the second grating is shifted by half a period, i.e., $100 \mu \mathrm{m}$ in the $x^{\prime}$-direction. The focal length of the Fourier lens is $120 \mathrm{~mm}$. The illumination angle of the plane wave is described by the direction cosine $(\cos \alpha, \cos \beta, \cos \gamma)$. The simulation results are shown in Fig. 3. The figure in (a1) shows the diffraction orders when the plane wave is parallel to the optical axis, i.e., the illumination angle at $\alpha=\beta=90^{\circ}$ and $\gamma=0^{\circ}$. (a2) shows the new diffraction orders because of the lateral shifts of the self-images of the first grating, as the illumination angle is changed to $\alpha=88^{\circ}, \beta=90^{\circ}$ and $\gamma=2^{\circ}$. (a3) shows only a lateral shift of the diffraction orders in (a1) along the $y$-axis resulting from the change of $\beta$ and $\gamma$ with $2^{\circ}$. (b) shows the sum of the intensities i.e., fringes obtained with a number of illumination angles. The results in (c1) and (c2) are the intensities achieved with different illumination angles at $y=0$ and $x=0$, respectively. (c1) indicates that different angles $\alpha$ and $\gamma$ contribute differently to the intensities in the observation plane as $\beta=90^{\circ}$. (c2) shows obviously lateral shifts of the intensity along the $y$-axis if $\alpha=90^{\circ}$ but $\beta$ and $\gamma$ vary. (d1) and (d2) show the summed intensities at $y=0$ and $x=0$. Hence, we conclude that with the increase of the illumination angle range, the intensities in the observation plane increase and expand to a larger region in both $x$-direction and $y$-direction. Therefore, fringes appear. Moreover, the fringes become broader and obtain a triangular profile with the increase of the number of different illumination angles. This indicates that spatially incoherent illumination, especially stemming from many different illumination angles, should result in the typical Lau fringes. The mathematical description of the intensity distributions of
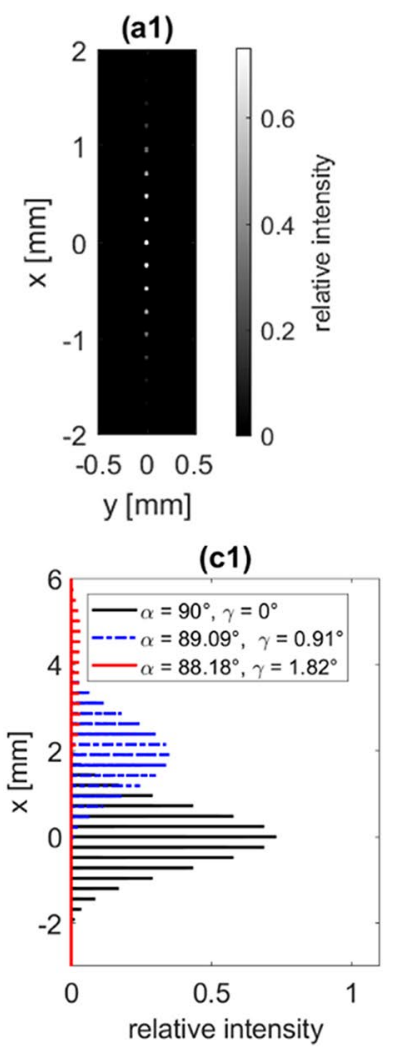

(a2)

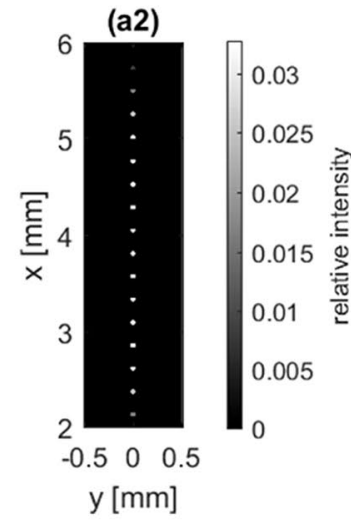

(c2)

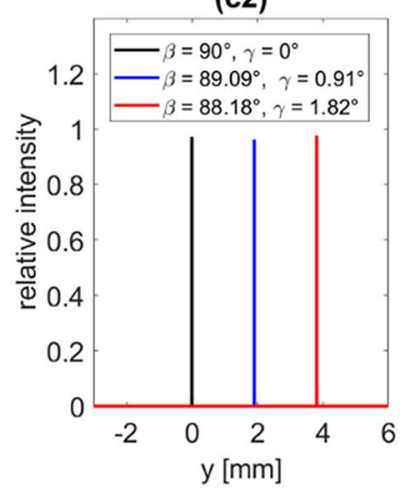

Fig. 3 Simulated intensity distributions. a1 Intensity image with plane wave parallel to the optical axis. a2 Intensity image with $\alpha=88^{\circ}, \beta=90^{\circ}, \gamma=2^{\circ}$. a3 Intensity image with $\alpha=90^{\circ}, \beta=88^{\circ}, \gamma=2^{\circ}$. b Intensity image with various illumination angles. This image shows the summed intensities $\alpha$ and $\beta$ separately vary from $90^{\circ}$ to $88^{\circ}$ with a step of $0.01^{\circ}$. c1 Intensities at $y=0$ as
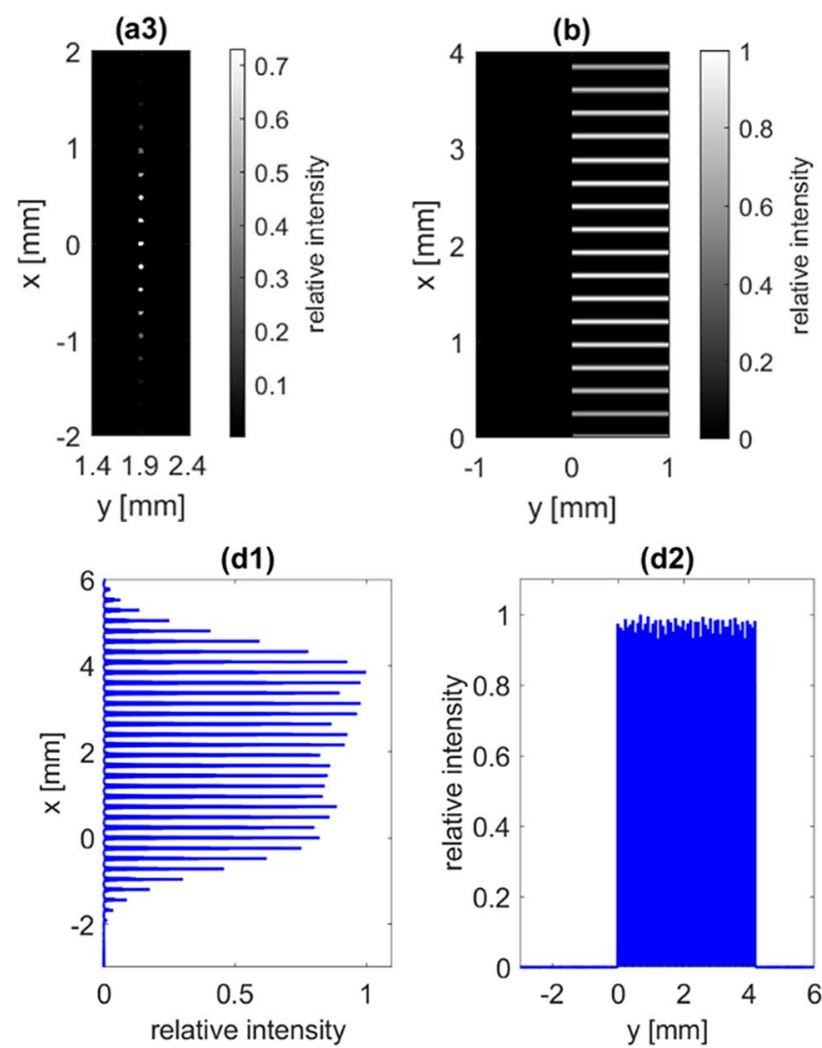

(d2)

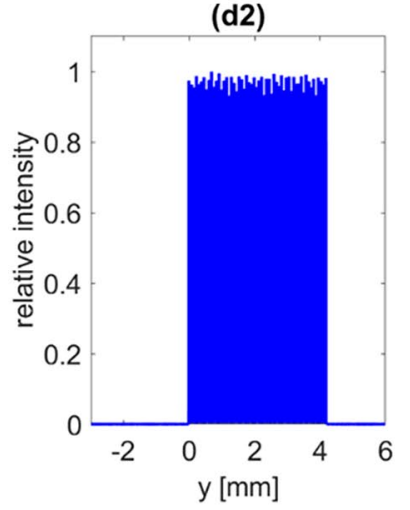

$\beta=90^{\circ}$ but with different $\alpha$ and $\gamma$. c2 Intensities at $x=0$ as $\alpha=90^{\circ}$ but with different $\beta$ and $\gamma$. d1 Intensities at normalized with the maximum in $y=0$ from $\mathbf{b}$. d2 Intensities at $x=0$ from $\mathbf{b}$ ). The intensities in $\mathbf{a 1}, \mathbf{a 2}, \mathbf{a 3}, \mathbf{b}$, and $\mathbf{c 1}$ are normalized with the maximum in $\mathbf{d 1}$. The intensities in $\mathbf{c 2}$ are normalized with the maximum in $\mathbf{d} \mathbf{2}$ 
these Lau fringes is shown in Eq. (6) [2, 29], where $\left(x_{1}^{\prime}, y_{1}^{\prime}\right)$ denotes the coordinate of any point on the first grating. The function $\hat{g}\left(x_{1}^{\prime}+z_{0} x / f, y_{1}^{\prime}+z_{0} y / f\right)$ denotes the Fresnel propagation of the second grating calculated at the distance $z_{0}$ and at the position $\left(x_{1}^{\prime}+z_{0} x / f, y_{1}^{\prime}+z_{0} y / f\right)$. The intensities $I_{\lambda}(x, y)$ are proportional to the cross correlation between the amplitude square of the first grating and the amplitude square of the Fresnel transform of the second grating.

$I_{\lambda}(x, y)=\frac{1}{\left(\lambda^{3} f d z_{0}\right)^{2}} \iint_{-\infty}^{\infty}\left|h\left(x_{1}^{\prime}, y_{1}^{\prime}\right)\right|^{2} \cdot\left|\widehat{g}\left(x_{1}^{\prime}+\frac{z_{0}}{f} x, y_{1}^{\prime}+\frac{z_{0}}{f} y\right)\right|^{2} \mathrm{~d} x_{1}^{\prime} \mathrm{d} y_{1}^{\prime}$

The numerical simulation results based on Eq. (6) are presented in Fig. 4, where both gratings are assumed to be infinite. The parameters of the two gratings and the Fourier lens are the same as for Fig. 3. In order to compare with the experimental results in Sect. 4, the wavelength of the illumination and the grating separation are set to be $405 \mathrm{~nm}$ and $96 \mathrm{~mm}$, respectively. The Lau fringes in Fig. $4 \mathrm{c}$ are periodic triangle distributed, which have a period around $250 \mu \mathrm{m}$ and are more uniform than the intensity distributions in Fig. 3d1. The Lau fringes are used in the optical lithography to produce analog structures. To this end, corresponding experiments in Sect. 4 will be carried out.

\section{Illumination concept with an LED array for Lau effect}

A UV-LED array [30] consisting of 7 UV-LEDs (Würth Eletronik eiSos $\mathrm{GmbH} \& \mathrm{Co}$. KG) is used as the light source in the setup of the Lau's experiment (Fig. 5a). Each UV-LED has a peak wavelength at $405 \mathrm{~nm}$, a spectral bandwidth of $15 \mathrm{~nm}$ and a wavelength range from 370 to $445 \mathrm{~nm}$ [30]. This broadband wavelength extends the range of the half of the Talbot distance of the self-images of the grating, i.e., $z_{0}$ in Sect. 3. This makes the slits of the self-image wider at a fixed $z_{0}$. As a result, the obtained Lau fringes using broadband wavelength are slightly broader than those using a single wavelength [29]. Each UV-LED has angular intensity distributions similar to a Lambertian light source, which emits light in all directions in front of the LED surface. The larger the angle between the light direction and the LED
Fig. 4 Simulated intensity distributions of Lau fringes. a Intensity image of Lau fringes. b Mean value of the relative intensities along $y$-axis from the intensity image. c Zoomed fringes in view of a region from $\mathbf{b}$ (a)

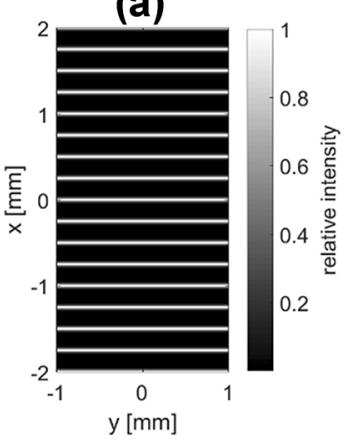

(a) Lau effect. a UV-LED array. b
Schematic setup. c Experimental setup with the UV-LED array for Lau effect. a UV-LED array. b
Schematic setup. c Experimen-

Fig. 5 Illumination concept (b)

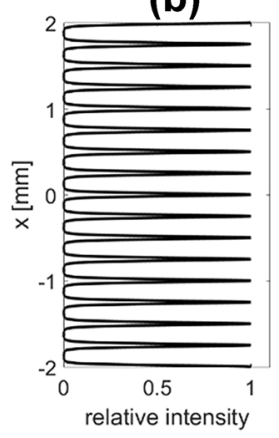

(c)

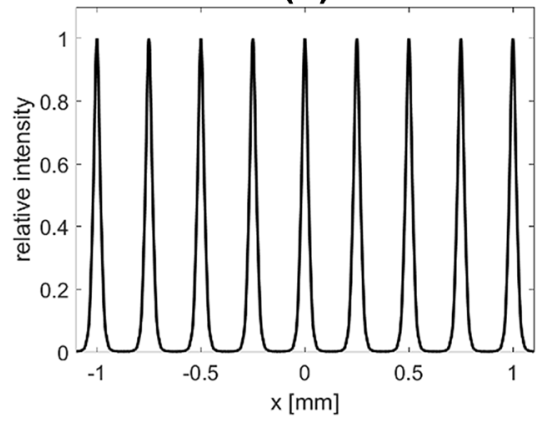

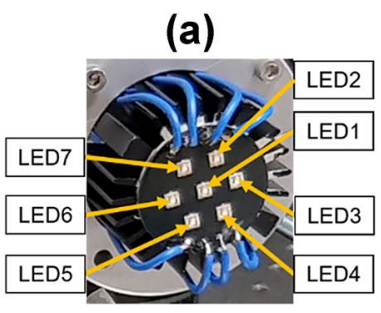

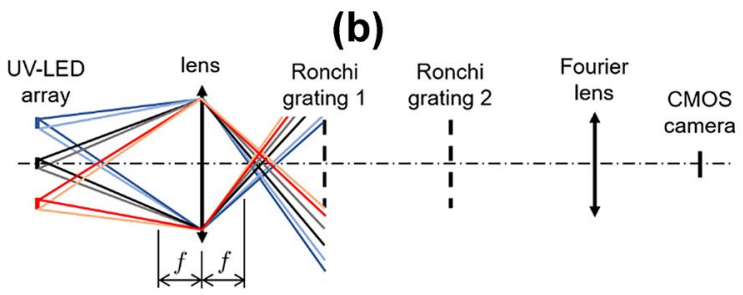

(c)

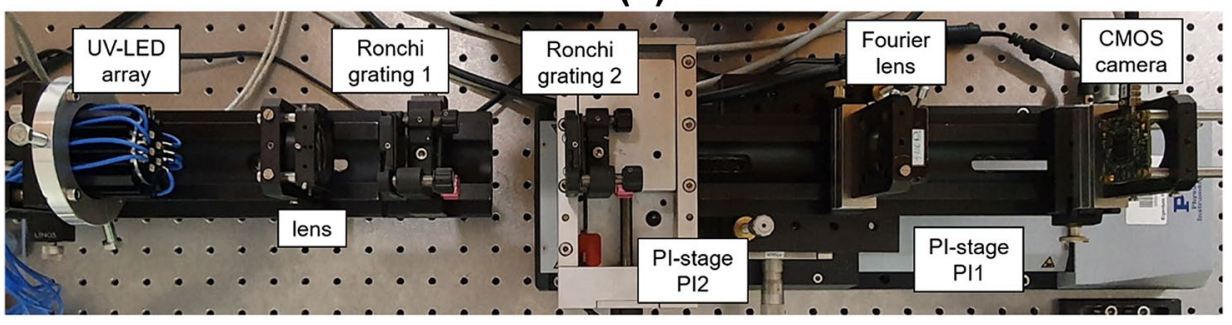


surface normal, the smaller is the light intensity. These angular intensity distributions will lead to the non-homogeneity of the illumination on the first grating. Subsequently, this results in the non-uniformity of the obtained Lau fringes. In order to improve the uniformity of the Lau fringes using the LED array, the distances between the centers of every two adjacent LEDs are identical, i.e., $14 \mathrm{~mm}$ for the experiment (Fig. 5). The radiant flux of each UV-LED is adjustable between 700 and $1100 \mathrm{~mW}$ by changing the electrical current of a power supply [30]. For the experiments in this section, we set a fixed electrical current around $240 \mathrm{~mA}$ for each UV-LED. Taking into account the conclusion in Sect. 3 that spatially incoherent illumination is necessary to produce Lau fringes, the illumination concept is designed as illustrated in Fig. 5b. The LED array is demagnified in an imaging step using a single lens $(f=27 \mathrm{~mm})$, so that the light behind the lens combines various illumination angles and the area of the source array is reduced. Furthermore, light from each UV-LED covers the whole surface of the first grating. This reduces the impact of the experimental tolerances on the summed intensity distribution on first grating, i.e., the angular intensity distribution of the LEDs, the positions of the LEDs, and the radiant flux of the LEDs. Therefore, the first grating is illuminated with spatially incoherent illumination with a relatively high optical power of light and a good homogeneity.

The experimental setup for the Lau effect is shown in Fig. 5c. The dimensions of the structures on each Ronchi grating are $20 \mathrm{~mm} \times 20 \mathrm{~mm}$. They both have period $p=200 \mu \mathrm{m}$ and mark-space ration $\delta=15 \%$. The illumination on the first grating includes light from infinite number of angles corresponding to $\alpha \in\left[47^{\circ}, 133^{\circ}\right]$ and $\beta \in\left[47^{\circ}, 133^{\circ}\right]$ (Fig. 2). The Fourier lens has the focal length $120 \mathrm{~mm}$. The automated PI-stage PI1 (Physik Instrumente (PI) GmbH \& Co. KG, M-521.DD) is used to move the elements behind the first grating axially so that the grating separation can be adjusted. In the following, the grating separation is set to be $96 \mathrm{~mm}$, which is approximately half of the Talbot distance of the first grating illuminated by the wavelength $405 \mathrm{~nm}$. The automated PI-stage PI2 (Physik Instrumente (PI) GmbH \& Co. KG, M-150.10) is applied to move the second grating laterally. The CMOS camera (IDS Imaging Development Systems GmbH, UI-3882LE-M) takes the intensity images in the observation plane.

First, the spatial coherence properties of the illumination are verified. In this experiment, the second grating is shifted laterally. The results in Fig. 6 show that with the lateral shift of the second grating, the Lau fringes also shift laterally. (a)

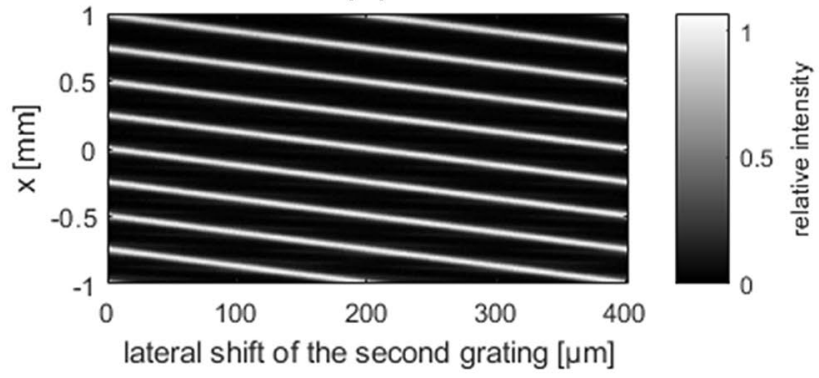

(b)

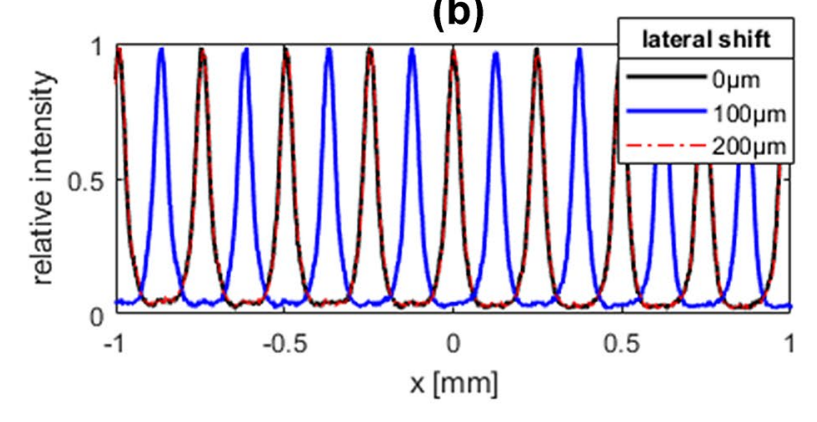

Fig. 6 Experimental results for verifying the spatial coherence properties of illumination with the UV-LED array. a Mean value of the intensities of Lau fringes along $y$-axis with lateral shifts of the second grating. b Relative intensity at different lateral shifts

Fig. 7 Captured intensity images. a Intensity image as only LED1 is turned on. b Intensity image as all seven of the LEDs are turned on. c Mean value of the intensities along $y$ -axis from intensity images, as we turn on only LED1, only LED2, only LED3 and all seven of the LEDs, respectively (a)

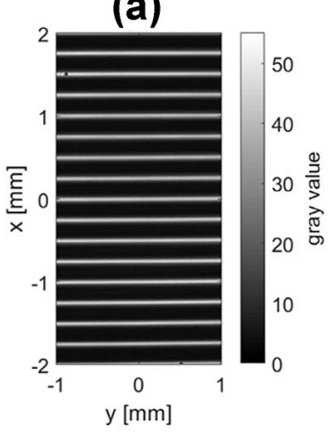

(b)

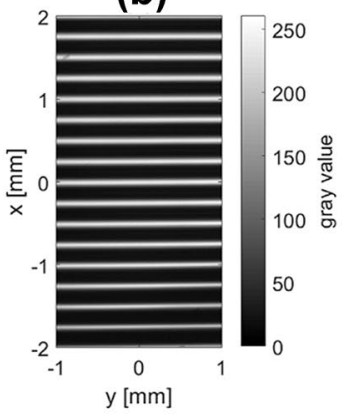

(c)

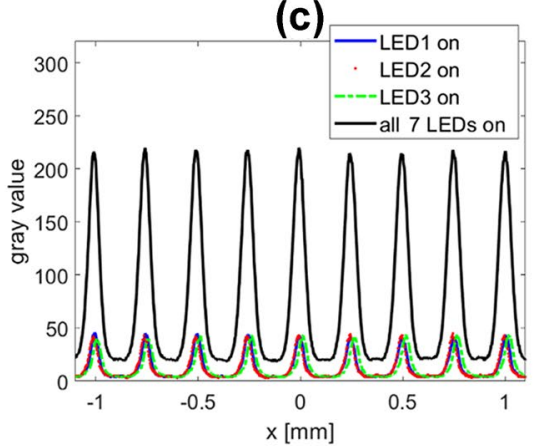


According to [4, 5], the illumination with the UV-LED array in our experimental setup is spatially incoherent.

In the second experiment, both PI1 and PI2 are fixed. The LEDs are turned on individually and at the same time, respectively. Figure 7a shows the captured intensity fringes when only LED1 is switched on. Figure $7 b$ shows the captured intensity fringes when all the LEDs are switched on. Figure $7 \mathrm{c}$ shows the mean values of the intensities along the $y$-axis when the exposure time of the CMOS camera is fixed. When all seven of the LEDs are turned on, the Lau fringes get their maximal intensities and are broader because of the additional illumination angles from the LEDs on the side. Compared with the simulated Lau fringes in Fig. 4 in Sect. 3, the Lau fringes from this experiment have the same period, i.e. $250 \mu \mathrm{m}$. Because of the achromatic wavelength used in the experiment, each Lau fringe is broader than the simulated Lau fringe, which is not so obvious. Moreover, due to the limited size of the two Ronchi gratings and the aperture stop, i.e., the Fourier lens in the experiment, the region of Lau fringes is also finite and their uniformity is not perfect as in the simulation results. The whole region filled with Lau fringes in the observation plane has a diameter of about $15 \mathrm{~mm}$, which is used for the optical lithography in Sect. 5.

\section{Application of Lau Fringes in Optical Lithography}

The Lau fringes obtained from the second experiment in Sect. 4 (Fig. 7) are used for lithography. There are two purposes. First, the possibility of using Lau fringes in the optical lithography to produce periodic triangular structures will be shown. Second, reducing exposure time by using an LED array instead of a single LED will be verified. For the second purpose, the cases when only LED1 is on and when all seven LEDs are on are investigated. Structures on glass wafers with a depth over $3 \mu \mathrm{m}$ will be lithographed. Besides, the development time will be fixed at $240 \mathrm{~s}$. When only LED1 is turned on, the detected optical power by the PowerMaxUSB sensor (Coherent Inc., 0264F11R) in the observation plane is about $1 \mu \mathrm{W}$. When all seven of the LEDs are turned on, the Lau fringes have an optical power about $7 \mu \mathrm{W}$. The applied photoresist and the developer are grayscale positive photoresist "ma-P 1275G" (micro resist technology GmbH) and "mr-D 526/S" (micro resist technology GmbH), respectively. In the exposure process of the lithography, the photoresist on the glass wafers reacts in the depth linearly to the intensity distribution of the Lau fringes. In the development process of the lithography, the exposed region of the photoresist will be removed, so that triangular distributed gaps will be observed.

Figure 8a1-c1 show parts of the lithographically structured profiles. They are measured with a white light interferometer (Veeco Instruments Inc., Wyko NT9300) and the pixel size for capturing the structures is set to $0.94 \mu \mathrm{m}$. The profiles of the periodic triangular distributed structures can be represented by their color bars and more precisely by the diagrams (a2), (b2), and (c2). The valleys of the profiles correspond to the peaks of the intensity distributions in Fig. 7. The exposure time using only the single LED1 for producing structures with a depth of around $3.05 \mu \mathrm{m}$ is $3600 \mathrm{~s}$ (Fig. 8b1, b2). The exposure time using the whole LED array for a depth of structures of around $3.4 \mu \mathrm{m}$ only takes $900 \mathrm{~s}$, which is one-fourth of $3600 \mathrm{~s}$. For structures with a depth over $3 \mu \mathrm{m}$, the LED array shows its advantage in saving the exposure time because of its higher optical power.

\section{Conclusions and Outlook}

In this paper, we have studied the application of the Lau fringes in optical lithography. The illumination concept of using an LED array has been proposed by taking into account the influence of the spatial coherence properties of the illumination, especially illumination angles, on creating Lau fringes. Due to its higher optical power, the LED array is superior to a single LED in saving the exposure time. The resolution of the lithographic structures is influenced by the optical system, the chemical reaction of the photoresist on the exposure dose. Of course, an LED with a much higher optical power can be also a solution to reduce the exposure time. However, the use of LED arrays helps us to understand the influence of the spatial coherence properties of illumination on Lau effect essentially. Furthermore, LED arrays provide more variety of illumination for optical systems. The image of an LED array with numerous LEDs instead of the first grating can be studied in future. Figure 9 shows an example of a structured illumination replacing the first grating. The structured illumination is a demagnified image of a rectangular LED array that consists of $5 \times 20$ LEDs. It will be interesting to study whether two-dimensional structures with this illumination can be obtained and be used in optical lithography. Moreover, the wafers used in the optical lithography can be located nonperpendicular to the optical axis to test whether is it possible to achieve blazed structures. 
(a1)

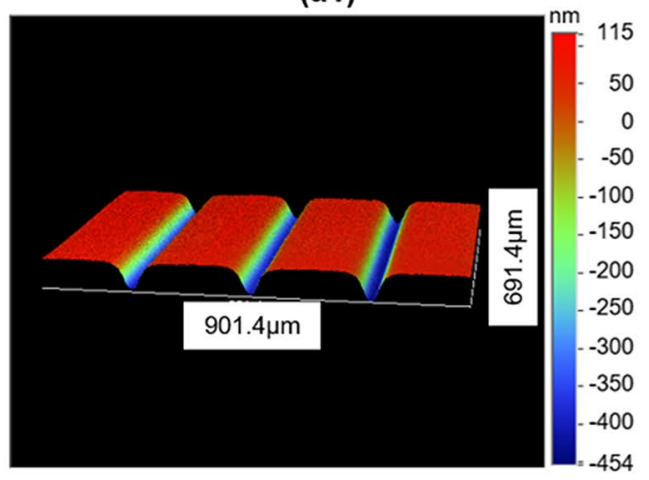

(b1)

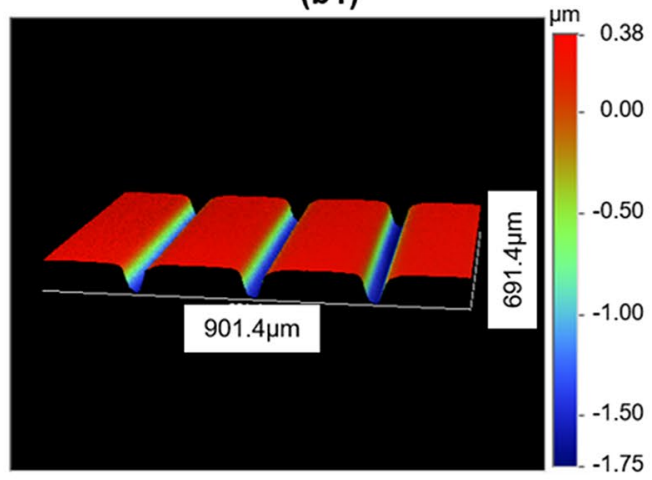

(c1)

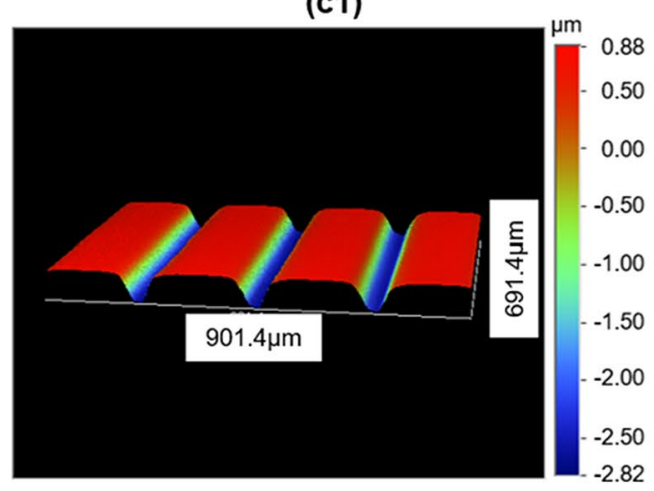

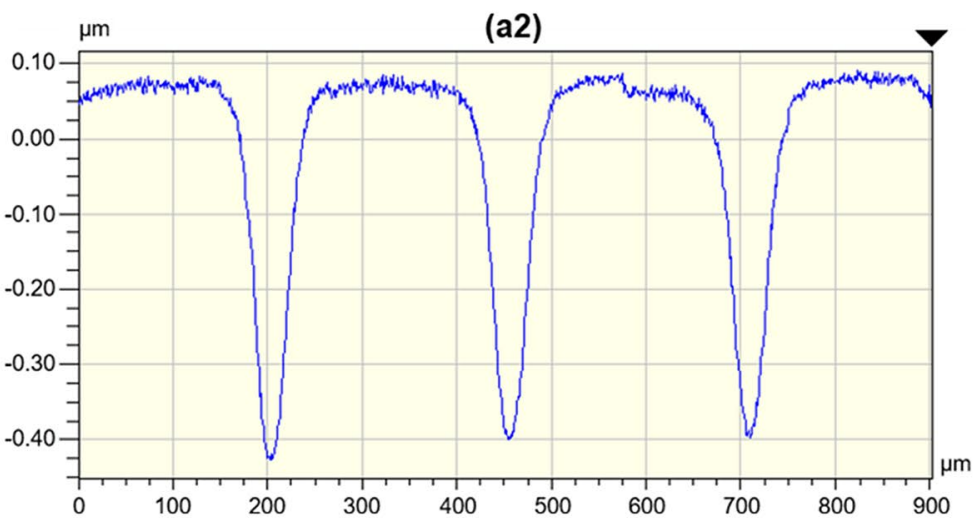

(b2)

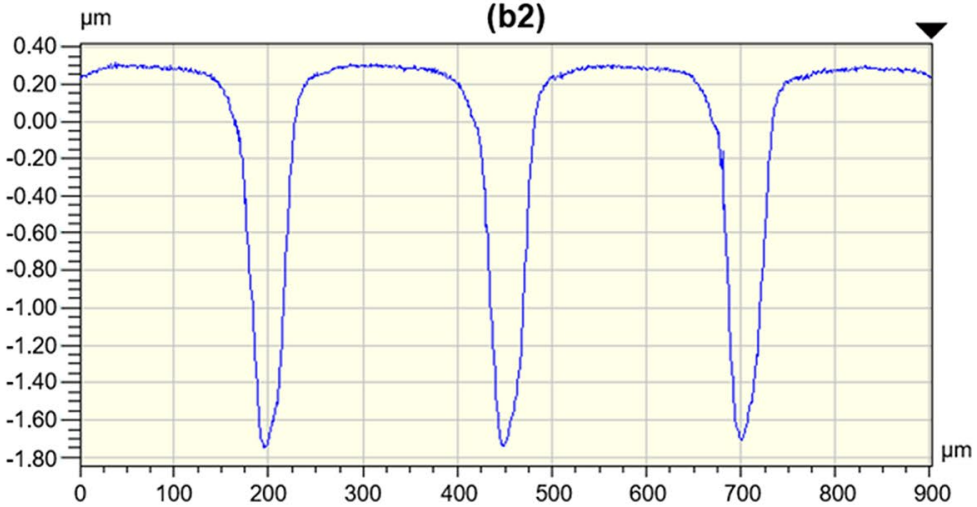

(c2)

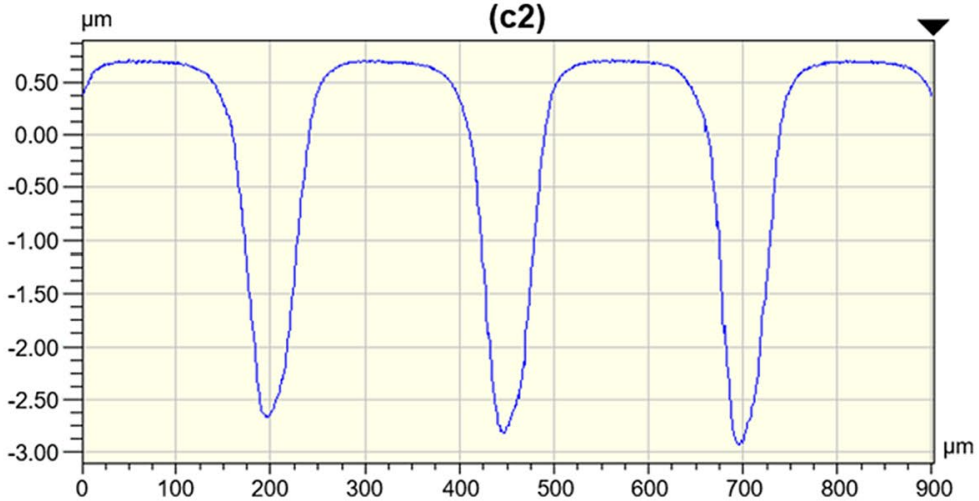

Fig. 8 Lithographically generated structures with the LED array and a single LED from the LED array. a1, a2 Only LED1 is turned on. The exposure time and development time are $900 \mathrm{~s}$ and $240 \mathrm{~s}$, respectively. b1, b2 Only LED1 is turned on. The exposure time and devel- opment time are $3600 \mathrm{~s}$ and $240 \mathrm{~s}$, respectively. c1, c2 All seven of the LEDs are turned on. The exposure time and development time are $900 \mathrm{~s}$ and $240 \mathrm{~s}$, respectively 
Fig. 9 Replacing the first grating with the image of an LED array

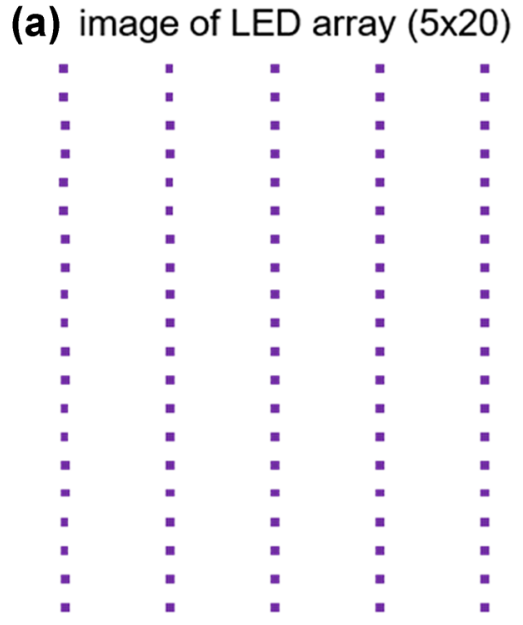

\section{(b) first grating}

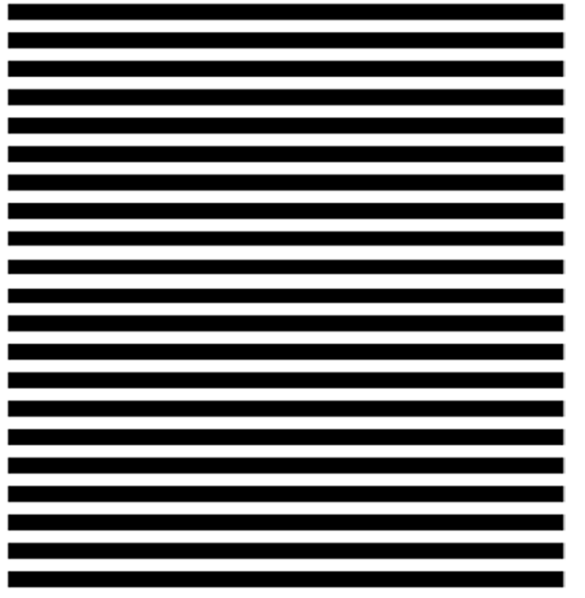

Acknowledgements We gratefully acknowledge the support by the Deutsche Forschungsgemeinschaft (DFG) in the framework of Research Training Group "Tip and laser-based 3D-nanofabrication in extended macroscopic working areas" (GRK 2182/1) at the Technische Universität Ilmenau, Germany. We thank Prof. J. Jahns for the suggestion of using LED arrays. We also thank Mr. T. Meinecke and Mr. D. Arsinio for help in constructing the UV-LED array.

Funding Open Access funding enabled and organized by Projekt DEAL.

\section{Disclosures}

Conflict of interest The authors declare no conflicts of interest.

Open Access This article is licensed under a Creative Commons Attribution 4.0 International License, which permits use, sharing, adaptation, distribution and reproduction in any medium or format, as long as you give appropriate credit to the original author(s) and the source, provide a link to the Creative Commons licence, and indicate if changes were made. The images or other third party material in this article are included in the article's Creative Commons licence, unless indicated otherwise in a credit line to the material. If material is not included in the article's Creative Commons licence and your intended use is not permitted by statutory regulation or exceeds the permitted use, you will need to obtain permission directly from the copyright holder. To view a copy of this licence, visit http://creativecommons.org/licenses/by/4.0/.

\section{References}

1. Lau E (1948) Beugungserscheinungen an Doppelrastern. Ann Phys 6:417

2. Jahns J, Lohmann AW (1979) The Lau effect (a diffraction experiment with incoherent illumination). Opt Commun 28:263-267

3. Sudol R, Thompson BJ (1981) Lau effect: theory and experiment. Appl Opt 20(6):1107-1116

4. Liu L (1988) Partially coherent diffraction effect between Lau and Talbot effects. J Opt Soc Am A 5(10):1709-1716
5. Tu J, Zhan L (1992) Analysis of general double periodic structure diffraction phenomena based on the ambiguity function. $\mathrm{J}$ Opt Soc Am A 9(6):983-995

6. Erickstad M, Gutierrez E, Groisman A (2015) A low-cost lowmaintenance ultraviolet lithography light source based on lightemitting diodes. Lab Chip 15(1):57-61

7. Bernasconi J, Scharf T, Vogler U, Herzig H (2018) High-power modular LED-based illumination systems for mask-aligner lithography. Opt Express 26:11503-11512

8. Ciou FY, Chen YC, Pan CT, Lin PH, Lin PH, Hsu FT (2015) Investigation of uniformity field generated from freeform lens with UV LED exposure system. Proc SPIE 9383:93830S

9. Wong AK (2001) Resolution enhancement techniques in optical lithography. SPIE, Bellingham

10. Chausse P, Le BE, Lis S, Shields P (2019) Understanding resolution limit of displacement Talbot lithography. Opt Express 27:5918-5930

11. Wolferen Hv, Abelmann L (2011) Laser interference lithography. In: Lithography: principles, processes and materials, pp 133-148

12. Hassanzadeh A, Mohammadnezhad M, Mittler S (2015) Multiexposure laser interference lithography. J Nanophotonics 9(1):093067

13. Delrot P, Loterie D, Psaltis D, Moser C (2018) Single-photon three-dimensional microfabrication through a multimode optical fiber. Opt Express 26:1766-1778

14. Gittard S, Nguyen A, Obata K, Koroleva A, Narayan R, Chichkov B (2011) Fabrication of microscale medical devices by two-photon polymerization with multiple foci via a spatial light modulator. Biomed Opt Express 2:3167-3178

15. Kurselis K, Kiyan R, Bagratashvili V, Popov V, Chichkov B (2013) 3D fabrication of all-polymer conductive microstructures by two photon polymerization. Opt Express 21:31029-31035

16. Isoyan A, Jiang F, Cheng Y, Cerrina F, Wachulak P, Urbanski L, Rocca J, Menoni C, Marconi M (2009) Talbot lithography: selfimaging of complex structures. J Vac Sci Technol B 27(6):2931

17. Weichelt T, Bourgin Y, Zeitner U (2017) Mask aligner lithography using laser illumination for versatile pattern generation. Opt Express 25:20983-20992

18. Pret A, Gronheid R, Engelen J, Yan P, Leeson M, Younkin T (2012) Evidence of speckle in extreme-UV lithography. Opt Express 20:25970-25978

19. Noordman O, Tychkov A, Baselmans J, Tsacoyeanes J, Politi G, Blahnik V, Maul M (2009) Speckle in optical lithography and 
its influence on linewidth roughness. J Micro/Nanolithography MEMS MOEMS 8(4):43002

20. Voelkel R, Vogler U, Bramati A, Weichelt T, Stuerzebecher L, Zeitner U.D, Motzek K, Erdmann A, Hornung M, Zoberbier R (2012) Advanced mask aligner lithography. In: Proc. SPIE 8326, Optical Microlithography, vol XXV, p 83261Y

21. Cao X, Feßer P, Fischer D, Hofmann M, Bourgin Y, Sinzinger S (2018) Der Lau-Effekt in der lithographischen Mikro-Nanostrukturierung. 119. DGaO-Jahrestagung, Aalen

22. García-Rodríguez L, Alonso J, Bernabéu E (2004) Grating pseudo-imaging with polychromatic and finite extension sources. Opt Express 12:2529-2541

23. Case W, Tomandl M, Deachapunya S, Arndt M (2009) Realization of optical carpets in the Talbot and Talbot-Lau configurations. Opt Express 17:20966-20974

24. Stuerzebecher L, Fuchs F, Harzendorf T, Zeitner U (2014) Pulse compression grating fabrication by diffractive proximity photolithography. Opt Lett 39:1042-1045

25. Testorf M, Jahns J, Khilo NA, Goncharenko AM (1996) Talbot effect for oblique angle of light propagation. Opt Commun 129:167-172

26. Saleh BEA, Teich MC (1991) Fundamentals of photonics. Wiley

27. Goodman JW (2005) Introduction to Fourier optics, 3rd edn. Roberts \& Co. Publishers

28. Schmidt JD (2010) Numerical simulation of optical wave propagation with examples in MATLAB. SPIE, Bellingham, Washington

29. Cao X (2020) Innovative Beleuchtung für neuartige Abbildungsund Lithographiesysteme. Dissertation, TU Ilmenau

30. Arsinio D (2019) Construction of UV-LED array. Project thesis, TU Ilmenau

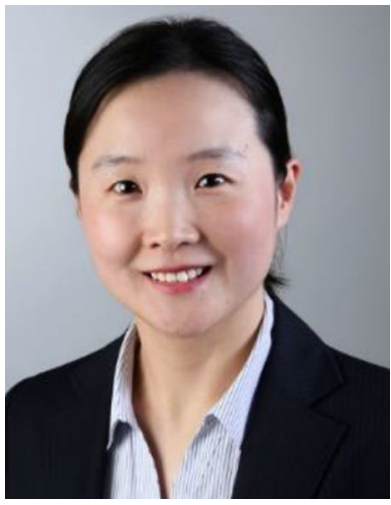

Xinrui Cao was born in Shandong, China. She received from the Technische Universität Ilmenau her MSc degree in Mechanical Engineering in 2014 and her Dr.Ing. degree in 2020. From April 2017 to March 2020, she was a member of the Research Training Group on 'Tip- and laserbased 3D-Nanofabrication in extended macroscopic working areas' funded through the German Research Foundation (DFG). Her research interest is illumination design for imaging and lithography systems.

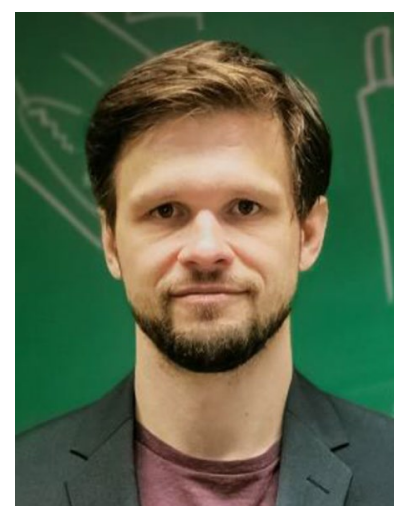

Patrick Feßer works since 2011 in the Department of Optical Engineering at the Technische Universität Ilmenau. He focuses on micro- and nanotechnology, lithography techniques, Plasma etching, characterization of optical elements.

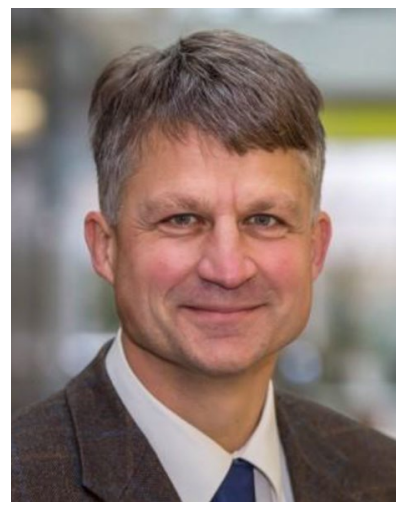

Stefan Sinzinger received his Dipl.-Phys. and Dr. degrees from the Friedrich-Alexander Universität Erlangen-Nürnberg, Institute for Applied Optics (Prof Dr A.W. Lohmann) in 1989 and 1993, respectively. In 2002, he became Professor for Optical Engineering ('Technische Optik') at the Technische Universität Ilmenau. Among numerous publications in international journals and conferences, Stefan Sinzinger is the co-author of the textbook 'Microoptics' and editor of the textbook 'Optical Information Processing' (author A.W. Lohmann). His current research focuses on the design, integration, fabrication, and application of (micro-) optical elements and hybrid optical (micro-) systems for innovative imaging and illumination. 